Full length article

\title{
The fracture toughness of eggshell
}

\author{
David Taylor*, Megan Walsh, Alexandra Cullen, Peter O’Reilly \\ Trinity Centre for Bioengineering, Trinity College Dublin, The University of Dublin, Dublin 2, Ireland
}

\section{A R T I C L E I N F O}

\section{Article history:}

Received 7 January 2016

Received in revised form 8 April 2016

Accepted 18 April 2016

Available online $\mathrm{xxxx}$

\section{Keywords:}

Egg

Shell

Eggshell

Toughness

Fracture

Defect

\begin{abstract}
A B S T R A C T
The shells of avian eggs are very brittle, but how brittle? Fracture toughness, $K_{c}$ is a standard measure used widely to characterise engineering materials. We devised a novel way to measure $K_{c}$ and applied it to commercial hens' eggs, obtaining a value of $0.3 \mathrm{MPa} \sqrt{ } \mathrm{m}$. This value is much lower than previous published values, which we argue are incorrect and misleading. We discuss how this exceptionally low toughness value (in comparison to that of other natural materials made from calcium carbonate) has been achieved by prevention of toughening mechanisms. Eggshell has an unusual combination of mechanical properties (low fracture toughness combined with high Young's modulus), making it ideally suited as a container for the developing chick, which must be stiff and rigid but also brittle enough to be broken when required. Further testing and analysis using the Theory of Critical Distances and Weibull probability theory allowed us to describe the effects of defects of various types: cracks, holes and notches, on the strength of whole eggs. These results are of commercial importance because many eggs break prematurely as a result of microscopic defects.
\end{abstract}

\section{Statement of Significance}

This paper presents the first accurate measurements of the fracture toughness of eggshell. These results are important because eggshell is a brittle material which fails from microscopic defects, so knowledge of the fracture toughness is essential to understand its mechanical performance.

The toughness value obtained is discussed in the context of other mechanical properties, of eggshell and other natural materials. This is useful for understanding how eggshell's stiffness and toughness make it ideally suited to its purpose, and the mechanisms by which toughness is achieved.

The paper also contains analysis of the effect of defect type, including cracks, notches and holes, to provide a fuller picture of defect tolerance which will be useful in the egg producing industry.

(c) 2016 Acta Materialia Inc. Published by Elsevier Ltd. All rights reserved.

\section{Introduction}

Avian eggshell is an extremely common biological material, which performs an important structural function. The egg must be sufficiently stiff and strong to protect the developing embryo, but during hatching the chick must be able to break out using its beak. The mechanical properties of eggshell are also of considerable commercial importance. Tens of millions of tonnes of eggs are produced annually, of which an estimated 8-10\% suffer damage during routine handling [1]; damaged eggs represent a considerable financial loss as well as a health hazard [2]. As a result, there has been a considerable body of work on the determination of eggshell structure and mechanical properties (for a recent review see [3]).

\footnotetext{
* Corresponding author.

E-mail address: dtaylor@tcd.ie (D. Taylor).
}

The sensitivity of a material to the presence of small cracks can be described using a mechanical property known as fracture toughness, which is commonly expressed in one of two different ways: (i) the energy required to propagate a crack by a given amount, $G_{c}$, given in units of energy per unit increase in the cracked area, and; (ii) a parameter $K_{c}$, which is related to the stress $\sigma_{\mathrm{f}}$ needed to propagate a crack of given length, $a$, according to the following Eq. (4):

$K_{c}=F \sigma_{f} \sqrt{\pi a}$

The constant $\mathrm{F}$ in this equation depends on the geometry of the crack and the body containing it, and the type of loading applied. These two toughness parameters are related to each other via the material's stiffness (Young's modulus, E), as follows [4]:

$K_{c}=\sqrt{G_{c} E}$ 
Classic brittle materials, such as glass and most ceramics, are relatively strong in compression but weak in tension, owing to low fracture toughness. When exposed to tensile stress, such a material is highly sensitive to cracks, and almost invariably fails at a stress lower than its inherent strength as a result of microscopic defects. Eggshell would appear to be such a material, and if so its $\mathrm{K}_{\mathrm{c}}$ value will be crucial in determining its mechanical performance.

Values of $K_{c}$ and $G_{c}$ for eggshell have been reported in three previous papers. Mabe et al. reported $K_{c}$ values of the order of 350 $\mathrm{Nmm}^{-3 / 2}$, which, when converted into SI units, gives $11.1 \mathrm{MPa} \sqrt{ } \mathrm{m}$ [5] (Note: the unusual conversion factor is due to the square root of length in the units for this quantity). They tested whole hens' eggs, compressing them between rigid, parallel platens, which caused failure by propagation of cracks from the contact points. They calculated $K_{c}$ using a formula previously devised by one of the authors [6]. More recently Xiao et al. reported similar values, of the order of 12.6 $\mathrm{MPa} \sqrt{ } \mathrm{m}$, in a paper investigating the effect of dietary supplements on hen egg properties [7]. Assuming a value of Young's modulus $\mathrm{E}=55 \mathrm{GPa}$ [1] gives values of $\mathrm{G}_{\mathrm{c}}$ (using Eq. (2) above) of $2.2 \mathrm{kJm}^{-2}$ and $2.6 \mathrm{kJm}^{-2}$ respectively for Mabe et al. and Xiao et al. Gosler et al. used a different method, obtaining $G_{c}$ by measuring the force needed to cut eggshell samples using scissors [8]. Their results, for Great Tit eggshells, show a lot of variability, but fall within the same order of magnitude as the results from the above workers, with values in the range $0.5-17 \mathrm{kJm}^{-2}$.

All of these values are much too high to be credible. For comparison, Table 1 lists some typical values of $K_{c}$ for a range of natural and manmade materials. Eggshell is a ceramic material, made largely from calcium carbonate in the form of calcite. There are almost no ceramic materials which have toughness values higher than $10 \mathrm{MPa} \sqrt{ } \mathrm{m}$. Pure mineral calcite has values in the range 0.2-1.8 MPa $\sqrt{ } \mathrm{m}[9,10]$. Some biological materials based on calcite (or the related mineral aragonite) have achieved higher toughness by evolving specialised microstructures (see below) but even these only reach values in the range 3-5 MPa $\sqrt{ } \mathrm{m}$. A further illustration of the impossibility of such high $K_{c}$ values comes from estimating the critical crack length, which can be done using Eq. (1) above and substituting the material's tensile strength for $\sigma_{\mathrm{f}}$. Using a typical

Table 1

Typical values of Fracture Toughness $K_{c}$ and Young's Modulus $E$ for Various Materials Data from [27] except the following: mussel shells [28]; nacre [17]; bone [18]; temporomandibular joint disc [29]; cartilage [30]; stratum corneum [31].

\begin{tabular}{lclc}
\hline Material & $E(\mathrm{GPa})$ & $\begin{array}{l}K_{c} \\
(\mathrm{MPa} \sqrt{ } \mathrm{m})\end{array}$ & $E / K_{c}\left(\mathrm{~m}^{-1 / 2} \times 10^{3}\right)$ \\
\hline $\begin{array}{lll}\text { Ceramics (natural and manmade) } \\
\text { Granite }\end{array}$ & 60 & 3 & 20.0 \\
Ice & 9.1 & 0.2 & 45.5 \\
Alumina & 390 & 4 & 97.5 \\
Silicon carbide & 440 & 3 & 146.7 \\
Concrete & 40 & 0.2 & 200.0 \\
Glass & 69 & 0.75 & 92.0 \\
Polymers and metals & & & \\
Perspex & 3.4 & 1.1 & 3.1 \\
Epoxy & 3 & 0.4 & 7.5 \\
Steel & 200 & 50 & 4.0 \\
Aluminium & 69 & 30 & 2.3 \\
Stiff biological materials & & & \\
Mussel shell & 80 & 3 & 26.7 \\
Nacre & 70 & 4.3 & 16.3 \\
Wood & 12 & 10 & 1.2 \\
Bone & 17 & 4 & 4.3 \\
Soft biological materials & & & \\
Temporomandibular joint disc & 0.0022 & 0.11 & 0.02 \\
Cartilage & 0.0075 & 0.08 & 0.04 \\
Stratum corneum & 0.013 & 0.007 & 1.86 \\
\hline
\end{tabular}

strength value of $15 \mathrm{MPa}[1,11]$ and the $\mathrm{K}_{\mathrm{c}}$ value from Mabe et al. we find a critical crack length of $0.34 \mathrm{~m}$. Since this is much larger than the size of an egg, it implies that the strength of eggs will never be reduced as a result of having cracks, which is contrary to all experience.

The aim of the present work was to devise a method to measure the fracture toughness of eggshell and, more generally, to assess the effect of small cracks and other defects such as holes on the strength of eggs.

\section{Methods and materials}

We used hens' eggs obtained from a retail outlet, described as "free range, size large". In total, 46 tests were carried out, of which 4 were discarded owing to errors (misalignment etc), giving 42 valid results. All eggs were used on the day of purchase. They were tested at room temperature $\left(18-21^{\circ} \mathrm{C}\right)$, after being kept for a minimum of one hour to equilibrate to the temperature of the laboratory. External dimensions were measured before testing: height $H$ in the long-axis direction and width $W$ defined as the maximum diameter normal to the long axis. Shell thickness $t$ was measured after testing, on a part of the fracture surface close to the crack initiation site. The contents were removed before testing by drilling a small hole in each end and blowing with compressed air. A hole of radius $a$ was drilled at half height using a high speed, low torque drill. Four different hole radii were used, varying from $0.25 \mathrm{~mm}$ to $2.5 \mathrm{~mm}$. The larger holes were created using successive drills of gradually increasing size; microscopic examination revealed no signs of cracking or damage caused by the drilling operation. In addition to these circular holes, sharp notches were created by first drilling holes and then extending them in the long-axis direction using either a file or a scalpel. This allowed us to create notches with lengths varying from $0.84 \mathrm{~mm}$ to $3.25 \mathrm{~mm}$ and root radii varying from $0.01 \mathrm{~mm}$ to $0.23 \mathrm{~mm}$ (see Fig. 1).

We devised a novel testing procedure which relies on the fact that, if a thin-walled sphere is loaded in axial compression, a simple biaxial stress state arises near its equator. A test rig was designed with the aim of applying axial compression in such a way as to avoid failure occurring at the loading points (see Fig. 2). Loading between flat metal plates, as carried out by other workers $[5,7]$ creates high local stresses at the contact points. To
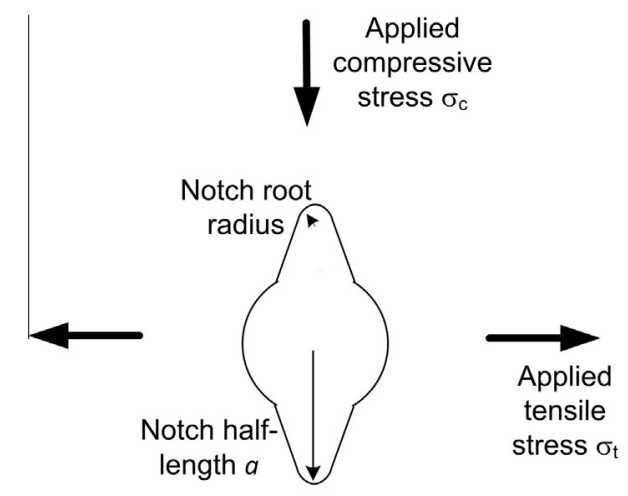

Fig. 1. Holes (of radius $a$ ) were drilled into whole eggs. Some holes were extended by machining to produce sharp notches (of half-length $a$ and root radius $\rho$ ). Under an applied remote compressive load the local stress field in the vicinity of the hole/ notch is biaxial, consisting of equal tensile and compressive stresses. 
prevent this we used hemispherical cups (actually wooden egg cups) and placed a layer of low-stiffness foam between the egg and the cup to redistribute the contact forces over a large area. We used a polyurethane foam packaging material. The inner diameter of the wooden hemispheres was $40 \mathrm{~mm}$; the undeformed thickness of the foam was $8 \mathrm{~mm}$. Typical egg dimensions were $\mathrm{H}=60 \mathrm{~mm}, \mathrm{~W}=45 \mathrm{~mm}$. As a result, when an axial compressive force was applied, the middle third of the egg was left exposed whilst the cups and foam covered the upper and lower thirds. An Instron testing machine was used to apply axial compression at a rate of $2 \mathrm{~mm} / \mathrm{min}$. Failure occurred by brittle fracture, i.e. the propagation of one or more cracks, accompanied by a sudden drop in the measured force.

The nominal stress at the location of the hole or notch was calculated assuming the egg to be a perfect sphere of radius $b$ subjected to an axial compressive force $F$. The overall stress pattern is complex [12] but at locations remote from the loading points it simplifies to a compressive stress $\sigma_{\mathrm{c}}$ in the axial direction and an equal and opposite tensile stress $\sigma_{t}$ in the circumferential direction [13], as shown in Fig. 1. At the equator (where our holes and notches were placed) these stresses are given by:

$\sigma_{t}=-\sigma_{c}=\frac{F}{2 \pi b t}$

Fracture toughness was calculated assuming that the sharp notches were equivalent to cracks of the same half-length, $a$ in this case being defined as the distance from the centre of the notch to its tip. Since our notches were oriented in the axial direction they experienced $\sigma_{t}$ as a normal tensile stress. $K_{c}$ can be calculated assuming that the notch is a crack of the same length, using Eq. (1) above with the stress as given in Eq. (3) and the parameter $F=1$.

After failure, samples of the fracture surfaces were examined using a scanning electron microscope, having first been coated

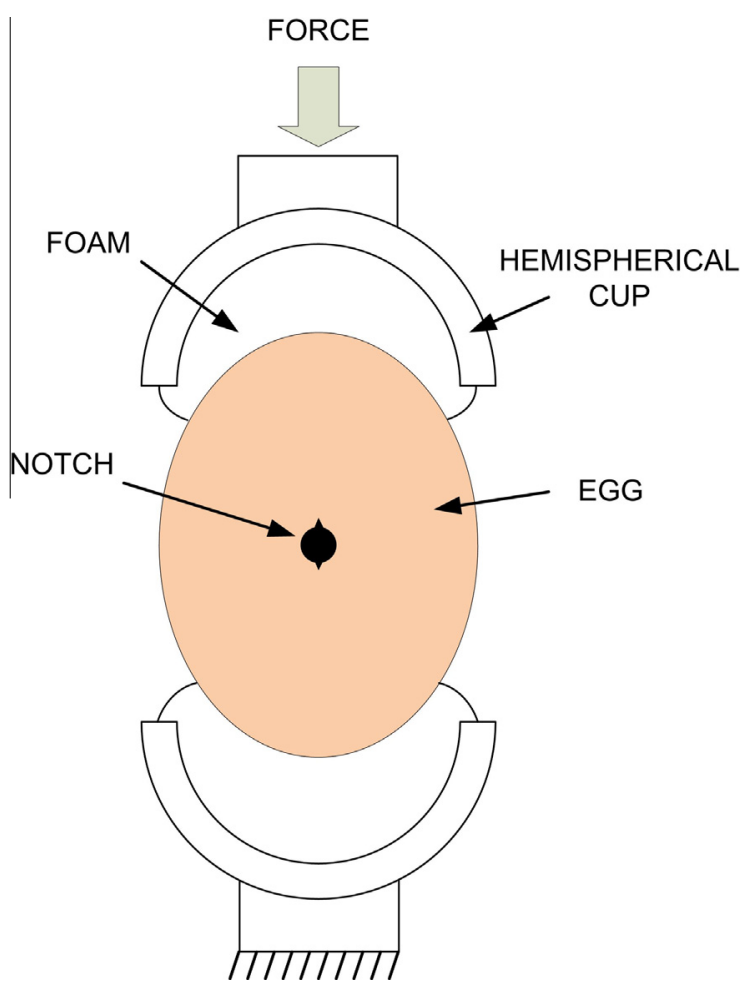

Fig. 2. A schematic of the test rig: the egg was contained in two wooden hemispheres, protected with a layer of foam material to prevent local stress concentrations. with a thin layer of gold to ensure electrical conduction. Statistical significance was investigated using the T-test with a threshold $\mathrm{p}$ value of 0.05 . Theoretical predictions were compared to experimental data by calculating $\mathrm{R}^{2}$ values.

\section{Results}

All samples failed by brittle fracture, i.e. by the formation and growth of cracks. Cracks grew from the holes and notches in all cases except the two smallest holes, those having radii $0.25 \mathrm{~mm}$ and $0.5 \mathrm{~mm}$, for which cracking started from the edge of one of the foam-covered areas, presumably due to some local stress concentration in those areas. Fig. 3 shows all the results, plotting the failure stress $\sigma_{\mathrm{tf}}$ as a function of the size of the introduced feature, defined as the radius for holes and the half-length for notches. For both holes and notches, increasing size caused a decrease in the stress to failure, which was statistically significant $(p<0.05)$. In Fig. 4, the measured value of $K_{c}$ is plotted as a function of notch root radius. Strictly speaking, $K_{c}$ can only be obtained from a crack, i.e. a notch with zero root radius: this plot shows the value of $K_{c}$ obtained assuming zero root radius.

Fig. 5 shows several images of the fractured areas. At low magnification (Fig. 5a), the fracture surface showed a series of approximately flat planes which reveal the typical palisade structure of eggshell, which consists of a series of calcite structures oriented through-thickness [14]. The average width of these planes (measured perpendicular to the thickness of the shell) was $44 \mu \mathrm{m}$. At higher magnification (Fig. 5b), each plane was seen to be not perfectly flat, but rather made up of a series of smaller planes having the appearance of individual cleavage facets, of typical size 1$3 \mu \mathrm{m}$, along with some rougher regions. The cleavage facets in a given area tended to have the same orientation, suggesting a common underlying crystal structure. When viewed from the surface of the shell (Fig. 5c) the crack was, in general, remarkably straight, showing little tendency to deviate from side to side, and only very occasionally to develop branches (Fig. 5d).

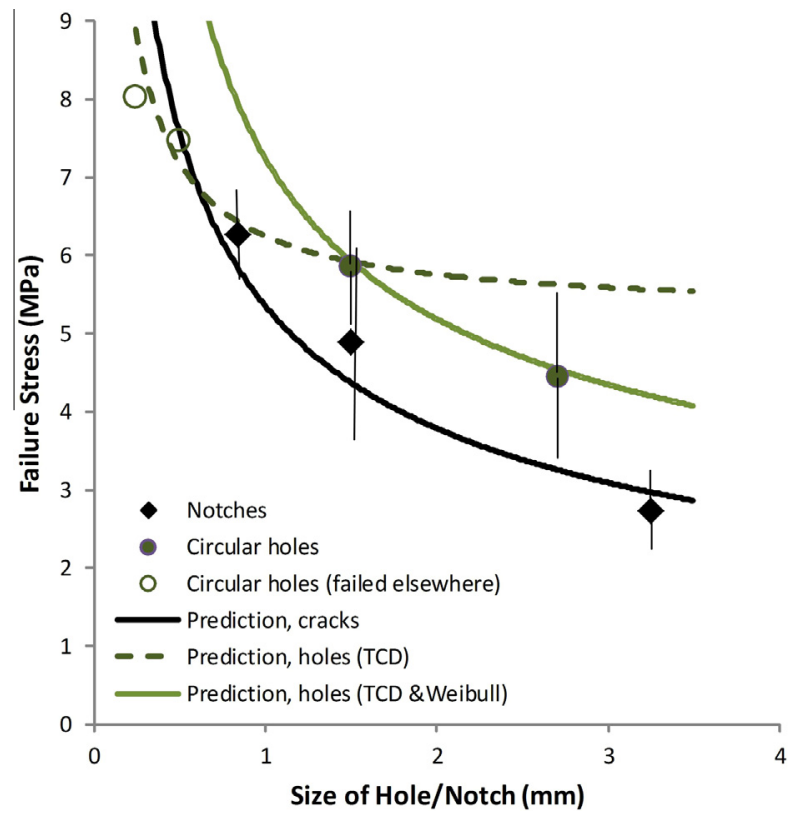

Fig. 3. Test results: applied tensile stress to failure $\sigma_{\mathrm{tf}}$ as a function of the size of the hole or notch $a$, (defined as the hole radius or notch half-length, see Fig. 1). Also shown are predictions for: sharp cracks (using the measured $K_{c}$ value); holes (using the TCD approach) and; holes (using TCD with a correction factor obtained using Weibull analysis). 


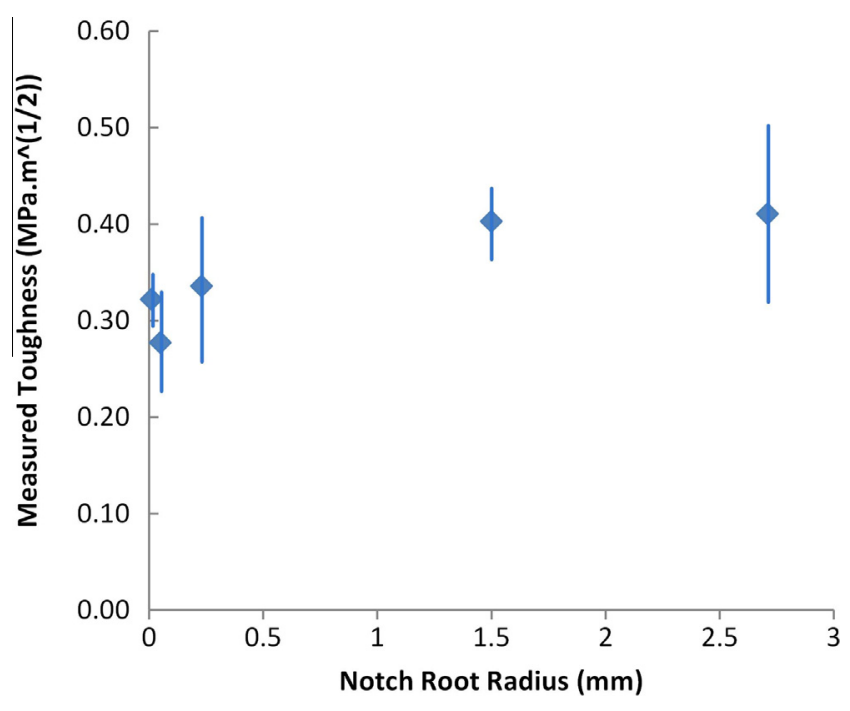

Fig. 4. Measured fracture toughness as a function of the root radius of the hole or notch. A valid $\mathrm{K}_{\mathrm{c}}$ value can be obtained by extrapolating the results to zero root radius.

\section{Discussion}

\subsection{The fracture toughness of eggshell and its biomechanical significance}

The value of $K_{c}$ can be estimated by extrapolating the results in Fig. 4 to a notch root radius of zero. This approach is commonly used to find the fracture toughness of brittle materials such as ceramics: ideally one would carry out a test on a sample containing a sharp pre-crack but it is difficult to introduce such cracks without fracturing the sample completely, so notches of various root radii are used instead [15]. Further confirmation of the validity of the result can be obtained by calculating a parameter known as the critical distance (see below).

In the present case the value obtained for $K_{c}$ was $0.3 \mathrm{MPa} \sqrt{ } \mathrm{m}$, with an estimation error of about $10 \%$. We believe this to be the first accurate estimate of the fracture toughness of eggshell. This is an extremely low value: as Table 1 shows it is less than the toughness of glass and much less than many other stiff biological materials such as bone. Some biological materials such as cartilage have even lower values, but these are soft, low-stiffness materials. Combining our result with other data shows that this material has a rather unusual combination of properties. Having a high Young's modulus of $55 \mathrm{GPa}$ [1], and a very low fracture toughness, eggshell has an exceptionally high value of the ratio $\mathrm{E} / \mathrm{K}_{\mathrm{c}}$ compared to other materials: Fig. 6 illustrates this, showing values of this parameter for various materials as a function of $E$. The only other materials which come close to having such a high value are certain engineering ceramics, including concrete and silicon carbide.

What is the biological significance of this ratio $\mathrm{E} / \mathrm{K}_{\mathrm{c}}$ ? The avian egg must be sufficiently rigid to protect the developing embryo, deforming minimally under the weight of the adult bird. This requirement is achieved partly by the egg's shape: (a thin-walled spherical structure has high rigidity for a given weight) and partly by using a material with a high Young's modulus. Eggshell has one of the highest Young's moduli of any natural material, being approximately three times greater than that of bone. The egg also needs to be reasonably strong, but high strength is not very important, because the weight of the adult bird will not give rise to very high stresses in the shell. In our tests, failure occurred at forces of the order $100-400 \mathrm{~N}$, equivalent to weights of about $10-40 \mathrm{~kg}$. If the egg falls out of the nest it may break on impact, but in these


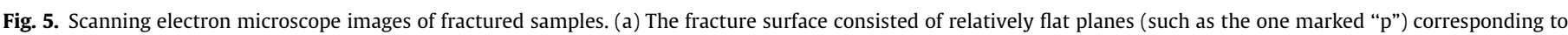

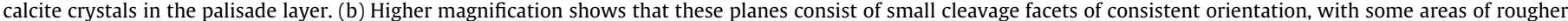

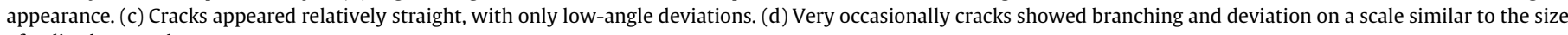
of palisade crystals. 


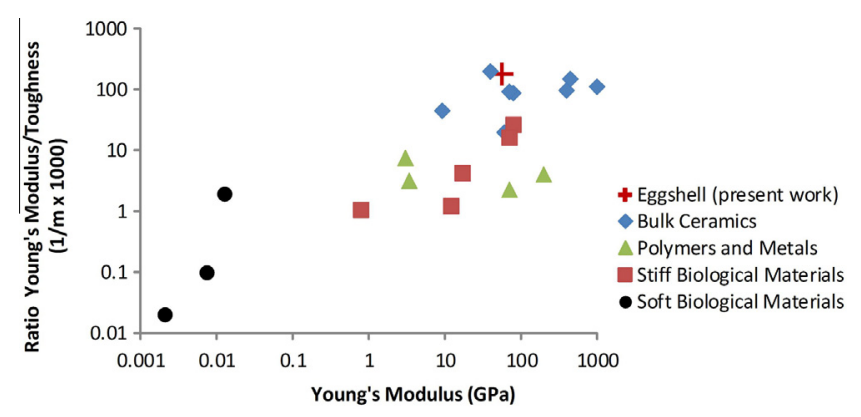

Fig. 6. Values of the ratio $E / K_{c}$ for various materials, plotted as a function of $E$.

circumstances eggs are almost always abandoned by the parents even when unbroken. In fact, the egg needs to be breakable, under the very particular circumstances in which the chick hatches out, using its beak to locally break a hole in the shell which it then enlarges. Just before hatching, the egg becomes thinner by erosion, making it easier to break. Low fracture toughness is also crucial to allow this process to occur given the relatively small forces which the chick can apply. Failure can be induced in a brittle material by making a hole which acts as a stress concentration: further stress induces a brittle fracture by crack propagation. Therefore, with a moderate strength, high Young's modulus and low fracture toughness eggshell is a material which is ideally suited to its purpose.

It is not entirely clear why previous workers (see above) obtained such impossibly high values of $\mathrm{K}_{\mathrm{c}}$ and $\mathrm{G}_{\mathrm{c}}$. The test used by Mabe et al. and reproduced by Xiao et al., in which the egg is compressed between rigid metal plates, is commonly used as a quality control test in the egg industry. However the mode of failure induced is very complex. The applied forces are transmitted through a very small contact area: under these conditions the local stress field near the contact area is complex and difficult to predict [12]. Cracks will initiate in or close to the contact area, and propagate away for a distance which depends on the applied force and the material's mechanical properties. Macleod et al. carried out a detailed simulation of this phenomenon using finite element analysis. They stopped short of attempting to estimate $K_{c}$, but commented that a value of the order of $0.2 \mathrm{MPa} \sqrt{ } \mathrm{m}$ would be consistent with their findings. The derivation of the formula for $K_{c}$ used by Mabe et al. (and subsequently by Xiao et al) is not explained in the paper. The quite different method used by Gosler et al., in which the energy is supplied by cutting with scissors, has successfully been applied to measure toughness in other materials. It may however not be suitable for very stiff, brittle materials which fail by cracking along cleavage planes, because the scissors may induce a lot of extra cracking events close to the main fracture path.

\subsection{Failure mechanisms in eggshell and other calcite/aragonite-based biological materials}

How does eggshell achieve this unusual combination of properties, in particular its very low toughness? It consists largely of calcium carbonate, in the form of calcite crystals orientated perpendicular to the shell surface, plus a small amount (less than $2 \%$ ) of organic material $[3,14]$. The fracture toughness of calcite mineral has been measured at various values, from 0.2 to $1.8 \mathrm{MPa} \sqrt{ } \mathrm{m}[9,10]$, being higher in material with low porosity and small grain size.

Some other biological materials, for example nacre, mussel shells and conch shells, have essentially the same composition, with calcium carbonate in either the calcite or aragonite forms. However these materials have fracture toughnesses which are ten times higher than that of eggshell (see Table 1). The remarkable toughness values of these materials have been the subject of much research in recent times (e.g. $[9,16,17])$. Structure at the micro and nano scale can give rise to toughening mechanisms, hindering crack growth. For example, nacre has a brick-and-mortar structure of rectangular crystals of thickness about $0.5 \mu \mathrm{m}$, separated by a very thin layer of organic material. This creates many weak interfaces which consume energy by debonding and cause a growing crack to deflect from side to side, greatly increasing the energy consumed in crack propagation. In bone, crack deflection occurs around longitudinal structures known as osteons, which have a width of typically $200 \mu \mathrm{m}$. These osteons often remain unbroken, giving rise to supporting ligaments behind the crack tip, which carry tensile stress and thus hinder further crack propagation $[8,19]$.

As Fig. 5 shows, these toughening mechanisms do not occur in eggshell. Though there exist structural units at the $100 \mu \mathrm{m}$ scale palisade columns which occupy most of the shell thickness [3] - it's clear that that they do not cause the crack to deflect around them. Cracks deviated only through small angles, and deviation rarely exceeded $5 \mu \mathrm{m}$ from a straight line (Fig. $5 \mathrm{c}$ ) except very occasionally (Fig. 5d) when the crack appeared to bifurcate around a palisade column. The fracture surface consisted of a series of approximately flat planes (Fig. 5a), which correspond to individual calcite crystals within the palisade columns. Microscopic cleavage facets within one of these planes had a consistent orientation, suggesting a single crystal of calcite (Fig. 5b). Apart from the cleavage facets, some other, rougher, areas can be seen in Fig. 5b, which may represent fracture through inclusions of organic material. Rodriguez-Navarro et al. showed that there is considerable crystallographic texture present, i.e. the crystals in the palisade layer tend to have a preferential orientation. They found that shells which showed greater variation in crystal orientation had higher strength [14]: this is most likely because the crack is forced to deviate slightly as it passes from one crystal to another, a minor toughening mechanism which may be responsible for raising $K_{c}$ to $0.3 \mathrm{MPa} \sqrt{ } \mathrm{m}$ above the minimum calcite value of $0.2 \mathrm{MPa} \sqrt{ } \mathrm{m}$.

\subsection{The defect tolerance of eggshell}

Knowing $K_{c}$ allows us to estimate the effect of cracks and similar sharp defects on the strength of eggs. Macleod et al. found that the strength of whole hens' eggs was, on average, 17.1 MPa when measured at the point of production but dropped to $14.8 \mathrm{MPa}$ when measured at a retail outlet [1]. Given our value of $K_{c}$ this implies that failure occurs from cracks of average length $2 a=0.20 \mathrm{~mm}$ at the production location and $0.26 \mathrm{~mm}$ at the retail location. This gives a sense of the very small defect sizes involved in practice and the challenges facing the industry in detecting and controlling such defects.

As Fig. 3 shows, the fracture mechanics equation (Eq. (1)) is able to give a reasonably good prediction of the data from the sharp notches. The $\mathrm{R}^{2}$ value in this case is 0.57 : in this case (given the scatter in the data) a perfect prediction would give an $R^{2}$ value of 0.65 , however this prediction is not expected to be perfect because the equation applies strictly only to perfect cracks. A major limitation of the $K_{c}$ parameter is that it only applies to cracks, i.e. notches having zero root radius. Other types of defect may occur, such as circular holes or notches with finite root radius. In order to predict the effect of these other kinds of stress concentration feature, and therefore to provide a more complete picture of the defect tolerance of eggshell, a different approach is required.

One such approach is known as the Theory of Critical Distances, TCD [20]. This approach requires an additional material property: a constant which is known as the critical distance, $L$. This constant can be used in various ways to predict failure by crack growth from 
a stress concentration feature. One approach, originally proposed to predict metal fatigue cracking [21] and subsequently applied to brittle fracture in a wide range of materials [20,22] is known as the Line Method. This involves calculating the average elastic stress along a line of length $2 L$, drawn from the point of maximum stress (e.g. the root of the notch) in the direction of crack propagation. Failure is predicted to occur if the value of this averaged stress is greater than or equal to a stress which, for brittle ceramic materials, is equal to the tensile strength of samples containing no stress concentration features [23].

In the present work we applied this approach to predict our results from samples containing circular holes. A circular hole subjected to a remote tensile stress $\sigma$ has a local stress field which can be estimated using Airy functions [24]. The appropriate equation, for the stress $\sigma_{\theta}$ (defined as circumferential with respect to the hole) at a location defined by angle $\theta$ (measured around the circle) and distance $r$ (measured from the circle's edge) is:

$\sigma_{\vartheta}=\frac{\sigma}{2}\left(1+\frac{a^{2}}{r^{2}}\right)-\frac{\sigma}{2}\left(1+3 \frac{a^{4}}{r^{4}}\right) \cos 2 \theta$

Solving for the present case in which there are two remote stresses $\sigma_{\mathrm{c}}$ and $\sigma_{\mathrm{t}}$, of equal magnitude and opposite sign, applied at right angles to each other, gives the following result for the cases

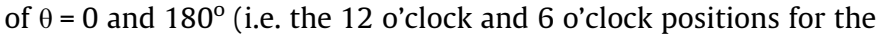
orientation shown in Fig. 1):

$\sigma=\sigma_{t}\left[1+3\left(\frac{a}{a+r}\right)^{4}\right]$

This stress has a maximum value of $4 \sigma_{t}$ and decreases with increasing distance from the hole, $r$. To predict $\sigma_{\mathrm{tf}}$ using the TCD (Line Method), we integrate this function to find the average stress between $r=0$ and $r=2 L$, putting this equal to the material's strength, $\sigma_{0}$. The result is:

$\sigma_{t f}=\frac{\sigma_{0}}{\left[1+\frac{a}{2 L}\left(1-\frac{1}{(1+2 L / a)^{3}}\right)\right]}$

Given that this approach applies to all stress concentration features, and therefore should apply also to cracks, it is possible to derive a relationship between the three material constants $L, \sigma_{o}$ and $K_{c}[25]$ as follows:

$L=\frac{1}{\pi}\left(\frac{K_{c}}{\sigma_{o}}\right)^{2}$

Since we already know $K_{c}$ for eggshell, the only unknown parameter is $\sigma_{0}$. This is the tensile strength of material containing no defects, but this is difficult to obtain experimentally because eggs almost always contain small defects. Previous workers [11] reported an average strength of $15.2 \mathrm{MPa}$ for whole eggs tested to failure by internal pressure, which is in agreement with results in the range 14.8-17.2 reported by Macleod et al. However another study [26] found a significantly higher value of 27.3 MPa when the stress was highly localised by loading sections of shell between pairs of rings. This shows that the result obtained for the tensile strength depends on the volume of material being stressed: smaller volumes will be stronger because they have less chance of containing large defects.

In the present case, the choice of a value of $21 \mathrm{MPa}$ for $\sigma_{\mathrm{o}}$ gives a perfect prediction for the hole of radius $1.5 \mathrm{~mm}$ (see Fig. 3) but overpredicts the strength of the larger hole. The $\mathrm{R}^{2}$ value for this prediction is very low at 0.16 , compared to the result for a perfect fit which would be 0.61 . Entwistle and colleagues found that the results in their two papers could be reconciled by using a Weibull probabilistic approach. This approach is commonly used to predict the strength of brittle materials which fail as a result of small, often undetectable, defects. The measured strength is expected to be proportional to the function $V_{s}^{1 / \mathrm{m}}$ where $V_{s}$ is the volume of material under stress and $m$ is the Weibull modulus, which can be obtained by analysing scatter in measured strength data. Macleod et al. [1] reported a Weibull modulus of 5.5 for eggshell.

Failure of a specimen containing a circular hole will occur from the highly-stressed region close to the hole surface: the volume of this region will be proportional to the square of the hole radius. We can expect that failure will be easier from larger holes, the failure stress being reduced by a factor $\mathrm{r}^{2 / \mathrm{m}}$. As Fig. 3 shows, if we apply this as a correction factor to our previous TCD approach, we obtain accurate predictions for both holes tested. The $\mathrm{R}^{2}$ value for this prediction is 0.59 , very close to the result of 0.61 for a perfect fit. We also tested two smaller hole sizes, $0.25 \mathrm{~mm}$ and $0.5 \mathrm{~mm}$ radius, but in these cases failure occurred not at the hole but elsewhere on the sample, as a result of limitations in the test rig, so these are obviously not valid results. Our prediction (using the TCD and Weibull) gives values of $17.0 \mathrm{MPa}$ and $10.7 \mathrm{MPa}$ for the $0.25 \mathrm{~mm}$ and $0.5 \mathrm{~mm}$ holes respectively. This implies that a $0.5 \mathrm{~mm}$ hole would reduce the strength of a whole egg (below the values of $15-17 \mathrm{MPa}$ reported by other workers) whilst the $0.25 \mathrm{~mm}$ hole would not reduce strength. Interestingly [11] found that a $0.4 \mathrm{~mm}$ radius hole did not reduce strength in their tests. We would predict a slight reduction, to $12.3 \mathrm{MPa}$, but the difference is small, so their result is broadly in agreement with ours.

Using Eq. (8) with the results now obtained for $K c$ and $\sigma_{o}$ gives a value for $L$ for eggshell of $0.065 \mathrm{~mm}$. This further justifies our use of sharply-notched samples to measure fracture toughness: it can be shown that a notched sample will yield values of $\mathrm{K}_{\mathrm{c}}$ similar to those obtained from a sharp crack provided the root radius of the notch is less than $L[20]$. Our two sharpest notches fulfilled this condition (see Fig. 5).

Our work had some limitations. We used only one source of eggs - hens' eggs from a retail outlet. This was satisfactory for our purpose, which was to develop an accurate method to measure fracture toughness, which can now be applied in future work to study the effect of variables such as species, time since laying, diet and the presence and developmental stage of the embryo. There is an organic membrane on the inside of the shell which, though it will not itself be strong enough to affect the results, may alter the state of hydration of the eggshell material: this was not investigated in the present work. For the purpose of stress analysis we assumed that the egg had a spherical shape. This assumption has been used in previous work (e.g. Macleod et al. [1]); in fact the egg shape does not deviate very much from a sphere: in our case the average height was larger than the average width by just $30 \%$. We used Eq. (3) which applies to the case of point forces at the two ends, whilst in our case the applied forces were distributed using foam material. This should not significantly affect the stresses in the vicinity of the notch because it is remote from the loading points. A more accurate stress analysis, taking these two issues into account, could be carried out using finite element analysis: though beyond the scope of the present paper this would be a useful piece of future work. We could not induce failure from defects as small as $0.5 \mathrm{~mm}$ or less owing to limitations of our loading method. It would be very useful to be able to do so, because the extrapolation of our theoretical predictions to submillimetre sizes is not necessarily reliable and would require experimental verification. This may be achievable in the future by modifications to the test rig.

\section{Conclusions}

1. The fracture toughness of eggshell can be measured by compressing whole eggs containing machined notches. 
2. The fracture toughness of free range hens' eggs was measured at $0.3 \mathrm{MPa} \sqrt{ } \mathrm{m}$. Eggshell has a remarkably high value of the ratio $E / K_{c}$, which makes it well fitted for its purpose.

3. This toughness value is close to the minimum measured for mineral calcite. This has been achieved by making eggshell from relatively large crystals and avoiding the various toughening mechanisms seen in other natural materials based on calcium carbonate such as nacre.

4. Analysis using the Theory of Critical Distances and Weibull probability theory allows us to predict the effect of defects of various types: cracks, notches and holes, which will be useful in assessing eggshell quality for industrial purposes.

\section{Acknowledgements}

We are grateful to the Centre for Microscopy and Analysis, Trinity College Dublin, for assistance with the electron microscopy.

\section{References}

[1] N. Macleod, M.M. Bain, J.W. Hancock, The mechanics and mechanisms of failure of hens' eggs, Int. J. Fract. 142 (1-2) (2006) 29-41.

[2] W. Messens, K. Grijspeerdt, L. Herman, Eggshell penetration by Salmonella: a review, World's Poult. Sci. J. 61 (1) (2005) 71-86.

[3] S.E. Solomon, The eggshell: strength, structure and function, Br. Poult. Sci. 51 (Suppl. 1) (2010) 52-59.

[4] M. Janssen, J. Zuidema, R. Wanhill, Fracture Mechanics, Spon Press, London, UK, 2002.

[5] I. Mabe, C. Rapp, M.M. Bain, Y. Nys, Supplementation of a corn-soybean meal diet with manganese, copper, and zinc from organic or inorganic sources improves eggshell quality in aged laying hens, Poult. Sci. 82 (12) (2003) 19031913.

[6] M.M. Bain, Eggshell Strength: A Mechanical/Ultrastructural Evaluation (Ph.D. thesis), University of Glasgow, UK, 1990. 1-266.

[7] J.F. Xiao, Y.N. Zhang, S.G. Wu, H.J. Zhang, H.Y. Yue, G.H. Oi, Manganese supplementation enhances the synthesis of glycosaminoglycan in eggshel membrane: a strategy to improve eggshell quality in laying hens, Poult. Sci. 93 (2) (2014) 380-388.

[8] A.G. Gosler, O.R. Connor, R.H.C. Bonser, Protoporphyrin and eggshell strength: preliminary findings from a passerine bird, Avian Biol. Res. 4 (4) (2011) 214 223.

[9] J. Lv, Y. Jiang, D. Zhang, Structural and mechanical characterization of Atrina pectinata and freshwater mussel shells, J. Bionic Eng. 12 (2) (2015) 276-284.

[10] L. Vavro, K. Sou-ìek, Study of the effect of moisture content and bending rate on the fracture toughness of rocks, Acta Geodyn. Geomater. 10 (2) (2013) $247-$ 253.
[11] K.M. Entwistle, T.Y. Reddy, The fracture strength under internal pressure of the eggshell of the domestic fowl, Proc. R. Soc. London B 263 (1369) (1996) 433438.

[12] R.D. Gregory, T.I. Milac, F.Y.M. Wan, Thick hollow sphere compressed by equal and opposite concentrated axial loads: an asymptotic solution, SIAM J. Appl. Mech. 59 (3) (1998) 1080-1097.

[13] M.G. Salvadori, Statics and Strength of Structures, Prentice Hall, New York, 1971.

[14] A. Rodriguez-Navarro, O. Kalin, Y. Nys, J.M. Garcia-Ruiz, Influence of the microstructure on the shell strength of eggs laid by hens of different ages, $\mathrm{Br}$. Poult. Sci. 43 (3) (2002) 395-403.

[15] K. Tsuji, K. Iwase, K. Ando, An investigation into the location of crack initiation sites in alumina, polycarbonate and mild steel, Fatigue Fract. Eng. Mater. Struct. 22 (1999) 509-517.

[16] F. Barthelat, H. Tang, P.D. Zavattieri, C.M. Li, H.D. Espinosa, On the mechanics of mother-of-pearl: a key feature in the material hierarchical structure, J. Mech. Phys. Solids 55 (2) (2007) 306-337.

[17] J.D. Currey, P. Zioupos, P. Davies, A. Casinos, Mechanical properties of nacre and highly mineralized bone, Proc. R. Soc. London B 268 (1462) (2001) 107111.

[18] R.K. Nalla, J.J. Kruzic, J.H. Kinney, R.O. Ritchie, Effect of aging on the toughness of human cortical bone: evaluation by R-curves, Bone 35 (6) (2004) 12401246.

[19] R.K. Nalla, J.S. Iken, J.H. Kinney, R.O. Ritchie, Fracture in human cortical bone: local fracture criteria and toughening mechanisms, J. Biomech. 38 (7) (2005) 1517-1525.

[20] D. Taylor, The Theory of Critical Distances: A New Perspective in Fracture Mechanics, Elsevier, Oxford, UK, 2007.

[21] H. Neuber, Theory of Notch Stresses: Principles for Exact Calculation of Strength With Reference to Structural Form and Material, 2., Springer, Verlag, Berlin, 1958, p. 292.

[22] J.M. Whitney, R.J. Nuismer, Stress fracture criteria for laminated composites containing stress concentrations, J. Compos. Mater. 8 (1974) 253-265.

[23] D. Taylor, Predicting the fracture strength of ceramic materials using the theory of critical distances, Eng. Fract. Mech. 71 (2004) 2407-2416.

[24] W.C. Young, R.G. Budynas, Roark's Formulas for Stress and Strain, seventh ed., McGraw-Hill, Boston, USA, 2002.

[25] D. Taylor, The theory of critical distances, Eng. Fract. Mech. 75 (2008) 16961705.

[26] K.M. Entwistle, H. Silyn-Roberts, S. Ochieng Abuodha, The relative fracture strengths of the inner and outer surfaces of the eggshell of the domestic fowl, Proc. R. Soc. London B 262 (1364) (1995) 169-174.

[27] M.F. Ashby, D.R.H. Jones, Engineering Materials, Pergamon, Oxford, UK, 1980

[28] S.C. Fitzer, W. Zhu, K.E. Tanner, V.R. Phoenix, N.A. Kamenos, M. Cusack, Ocean acidification alters the material properties of Mytilus edulis shells, J. R. Soc. Interface 12 (103) (2015).

[29] K. Koombua, R.M. Pidaparti, M.W. Beatty, Fracture toughness estimation for the TMJ disc, J. Biomed. Mater. Res. Part A 79 (3) (2006) 566-573.

[30] M.V. Chin-Purcell, J.L. Lewis, Fracture of articular cartilage, J. Biomech. Eng. 118 (4) (1996) 545-556.

[31] K.S. Wu, W.W. Van Osdol, R.H. Dauskardt, Mechanical properties of human stratum corneum: effects of temperature, hydration, and chemical treatment, Biomaterials 27 (5) (2006) 785-795. 